International Journal of Pure and Applied Mathematics

Volume 89 No. 5 2013, 689-697

ISSN: 1311-8080 (printed version); ISSN: 1314-3395 (on-line version)

url: http://www.ijpam.eu

doi: http://dx.doi.org/10.12732/ijpam.v89i5.5

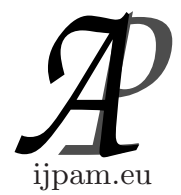

\title{
ON THE UNIVALENCE OF AN INTEGRAL OPERATOR
}

\author{
A. Selvam ${ }^{1}$, P. Sooriya Kala ${ }^{2}$, N. Marikkannan ${ }^{3} \S$ \\ ${ }^{1,2}$ Department of Mathematics \\ VHNSN College \\ Virudhunagar, 626001, India \\ ${ }^{3}$ Department of Mathematics \\ Government Arts College \\ Melur, 625106, INDIA
}

\begin{abstract}
Sufficient conditions for the univalence of an integral operator is obtained. Relevant connections of the results obtained with the earlier are also pointed out.
\end{abstract}

AMS Subject Classification: $30 \mathrm{C} 45,30 \mathrm{C} 80$

Key Words: analytic functions, univalent functions, Hadamard product, integral operators, Schwarz lemma

\section{Introduction}

Let $\mathcal{A}$ denote the class of functions of the form

$$
f(z)=z+\sum_{k=2}^{\infty} a_{k} z^{k} \quad\left(a_{k} \geq 0\right)
$$

which are analytic in the open disc

$$
\Delta=\{z \in \mathbb{C}:|z|<1\} .
$$

Let $\mathcal{S}$ be the class of functions $f \in \mathcal{A}$, which are univalent in $\Delta$.

Received: August 31, 2013

(c) 2013 Academic Publications, Ltd.

$\S$ Correspondence author 
For two functions $f_{j}(j=1,2)$ given by

$$
f_{j}(z)=z+\sum_{k=2}^{\infty} a_{k, j} z^{k} \quad(j=1,2),
$$

the Hadamard product or convolution, denoted by $\left(f_{1} * f_{2}\right)(z)$, given by

$$
\left(f_{1} * f_{2}\right)(z)=z+\sum_{k=2}^{\infty} a_{k, 1} a_{k, 2} z^{k}
$$

For complex numbers $\alpha_{1}, \alpha_{2}, \ldots, \alpha_{q}$ and $\beta_{1}, \beta_{2}, \ldots, \beta_{s} ;\left(\beta_{j} \in \mathbb{C} \backslash \mathcal{Z}_{0}^{-} ; \mathcal{Z}_{0}^{-}=\right.$ $\{0,-1,-2, \ldots\}$ for $j=1,2, . ., s)$, recently in [11] an operator $\mathcal{D}_{\lambda, \mu}^{m}\left(\alpha_{1}, \beta_{1}\right) f(z)$ : $\mathcal{A} \rightarrow \mathcal{A}$ is defined by

$$
\begin{aligned}
& \mathcal{D}_{\lambda, \mu}^{m}\left(\alpha_{1}, \beta_{1}\right) f(z)=z \\
& \quad+\sum_{k=2}^{\infty}[1+(k-1)(\lambda-\mu+k \mu \lambda)]^{m} \frac{\left(\alpha_{1}\right)_{k-1}\left(\alpha_{2}\right)_{k-1} \ldots\left(\alpha_{q}\right)_{k-1}}{\left(\beta_{1}\right)_{k-1}\left(\beta_{2}\right)_{k-1} \ldots\left(\beta_{s}\right)_{k-1}(k-1) !} a_{k} z^{k}
\end{aligned}
$$

where $q \leq s+1 ; q, s, m \in \mathbb{N}_{0}:=\mathbb{N} \cup\{0\} ; z \in \Delta$ and $\mathbb{N}$ denotes the set of all positive integers and $0 \leq \mu \leq \lambda \leq 1$. Also $(x)_{k}$ is the Pochhammer symbol defined in terms of gamma functions, as

$$
(x)_{k}=\frac{\Gamma(x+k)}{\Gamma(x)}=\left\{\begin{array}{lll}
1 & \text { if } & k=0 \\
x(x+1) \ldots(x+k-1) & \text { if } & k \in \mathbb{N}
\end{array}\right.
$$

For suitable values of $\alpha_{i^{\prime} s}, \beta_{j^{\prime} s}, q, s, m, \lambda$ and $\mu$ we can deduce several operators such as Sălăgean derivative operator [20], Ruscheweyh derivative operator [19], fractional calculus operator [13], Carlson-Shaffer operator [9], DziokSrivatsava operator [10] and also the operator introduced by Darus et al [1] and the operator introduced by Selvaraj et al [21].

Using the operator $\mathcal{D}_{\lambda, \mu}^{m}\left(\alpha_{1}, \beta_{1}\right) f(z)$ we now introduce the following. For $n \in \mathbb{N} \cup\{0\}$ and $\gamma_{1}, \gamma_{2}, \ldots, \gamma_{n}, \delta \in \mathbb{C} \backslash\{0,-1,-2, \ldots\}$ we define the integral operator

$$
\begin{aligned}
I_{\gamma_{i}, \delta}\left(\lambda, \mu, m ; \alpha_{1}, \beta_{1} ; z\right): \mathcal{A}^{n} & \rightarrow \mathcal{A} \text { by } \\
I_{\gamma_{i}, \delta}\left(\lambda, \mu, m ; \alpha_{1}, \beta_{1} ; z\right) & =\left\{\delta \int_{0}^{z} t^{\delta-1} \prod_{i=1}^{n}\left(\frac{\mathcal{D}_{\lambda, \mu}^{m}\left(\alpha_{1}, \beta_{1}\right) f_{i}(t)}{t}\right)^{\frac{1}{\gamma_{i}}} d t\right\}^{\frac{1}{\delta}} .
\end{aligned}
$$

This operator $I_{\gamma_{i}, \delta}\left(\lambda, \mu, m ; \alpha_{1}, \beta_{1} ; z\right)$ generalizes many operators which were introduced and studied by many authors. We list some of them. 
1. If $\mu=0$ the above integral operator reduces to

$$
F_{\gamma_{i}, \delta}\left(\lambda, m ; \alpha_{1}, \beta_{1} ; z\right)=\left\{\delta \int_{0}^{z} t^{\delta-1} \prod_{i=1}^{n}\left(\frac{\mathcal{D}_{\lambda}^{m}\left(\alpha_{1}, \beta_{1}\right) f_{i}(t)}{t}\right)^{\frac{1}{\gamma_{i}}} d t\right\}^{\frac{1}{\delta}} .
$$

This operator was introduced and studied by Selvaraj et al [22].

2. Let $\mu=0, q=2, s=1, \alpha_{1}=\beta_{1}, \alpha_{2}=1, \gamma_{i}=\frac{1}{\alpha_{i}}$ and $\delta=1$, this operator reduces to

$$
I\left(f_{1}, f_{2}, \ldots, f_{n}\right)(z)=\int_{0}^{z} \prod_{i=1}^{n}\left(\frac{D_{\lambda}^{m} f_{i}(t)}{t}\right)_{i}^{a} d t
$$

where $D_{\lambda}^{m}(f(z))$ is the well known Al-oboudi differential operator. This operator $I\left(f_{1}, \ldots, f_{n}\right)(z)$ was introduced and studied by S. Bulut [8].

3. For $m=0, q=2, s=1, \alpha_{1}=\beta_{1}, \alpha_{2}=1, \gamma_{i}=\frac{1}{\alpha-1}$ and $\delta=n(\alpha-1)+1$, then this operator reduces to

$$
F_{n, \alpha}(z)=\left[(n(\alpha-1)+1) \int_{0}^{z} \prod_{i=1}^{n}\left(f_{i}(t)\right)^{\alpha-1} d t\right]^{\frac{1}{n(\alpha-1)+1}} .
$$

Studied by D. Breaz and N. Breaz and H.M. Srivatsava [7].

4. Let $m=0, q=2, s=1, \alpha_{1}=\beta_{1}, \alpha_{2}=1, \gamma_{i}=\frac{1}{a_{i}}$ and $\delta=1$. Then this operator reduces to

$$
F_{\alpha}(z)=\int_{0}^{z} \prod_{i=1}^{n}\left(\frac{f_{i}(t)}{t}\right)^{a_{i}} d t
$$

which was introduced and studied by D. Breaz and N. Breaz [4].

5. Let $m=0, q=2, s=1, \alpha_{1}=2, \beta_{1}=1, \alpha_{2}=1, \gamma_{i}=\frac{1}{\alpha_{i}}$ and $\delta=1$ then the operator reduces to

$$
G_{\alpha}(z)=\int_{0}^{z} \prod_{i=1}^{n}\left(f_{i}^{\prime}(t)\right)^{\alpha_{i}} d t
$$

which was introduced and studied by D. Breaz et al [5].

Apart from this several well known and new integral operators will follow as a special case of $I_{\gamma_{i}, \delta}\left(\lambda, \mu, m ; \alpha_{1}, \beta_{1} ; z\right)$. 


\section{Preliminaries}

Theorem 2.1. (Schawarz Lemma) Let $f \in \mathcal{A}$ satisfy the condition $|f(z)|<1$ for all $z \in \Delta$, then

$$
|f(z)| \leq|z| \quad(z \in \Delta) .
$$

Equality holds for the functions $f(z)=c z$ where $|c|=1$.

Theorem 2.2. (see [15]) Let $\delta$ be the complex number with $\Re \delta>0$. If $f \in \mathcal{A}$ satisfies

$$
\frac{1-|z|^{2 \delta}}{\Re \delta}\left|\frac{z f^{\prime \prime}(z)}{f^{\prime}(z)}\right| \leq 1 \quad(z \in \Delta)
$$

then the function

$$
F_{\delta}(z)=\left[\delta \int_{0}^{z} t^{\delta-1} f^{\prime}(t) d t\right]^{\frac{1}{\delta}}=z+\ldots
$$

is analytic and univalent in $\Delta$.

Theorem 2.3. (see [16]) Let $\delta$ be a complex number and $\Re \delta>0$ and $c \in \mathbb{C}(|c|<1 ; c=-1)$. If $f \in \mathcal{A}$ satisfies

$$
\left.|c| z\right|^{2 \delta}+\left(1-|z|^{2 \delta}\right) \frac{z f^{\prime \prime}(z)}{\delta f^{\prime}(z)} \mid \leq 1 \quad(z \in \Delta),
$$

then the function

$$
F_{\delta}(z)=\left[\delta \int_{0}^{z} t^{\delta-1} f^{\prime}(t) d t\right]^{\frac{1}{\delta}}=z+\ldots
$$

is analytic and univalent in $\Delta$.

Theorem 2.4. (see [18]) Let $f \in \mathcal{A}$, satisfies the condition

$$
\left|\frac{z^{2} f^{\prime}(z)}{f^{2}(z)}-1\right| \leq 1, \quad(z \in \Delta) .
$$

Also let $\alpha \in\left[1, \frac{3}{2}\right]$ and $c \in \mathbb{C}$. If $|c| \leq \frac{3-2 \alpha}{\alpha}(c=-1)$ and $|f(z)| \leq 1(z \in \Delta)$ then the function

$$
H_{\alpha}(z)=\left(\alpha \int_{0}^{z}[g(t)]^{\alpha-1} d t\right)^{\frac{1}{\alpha}}
$$

belongs to $\mathcal{S}$. 


\section{3. main results}

Theorem 3.1. Let $\mathcal{D}_{\lambda, \mu}^{m}\left(\alpha_{1}, \beta_{1}\right) f_{i} \in \mathcal{A}$ for $i=1,2, \ldots, n$ and each satisfy (2.1). Also for $M \geq 1$, let $\gamma_{i}, \delta$ be complex numbers such that

$$
\Re \delta \geq \sum_{i=1}^{n} \frac{2 M+1}{\left|\gamma_{i}\right|} \quad \text { and } c \in \mathbb{C} .
$$

If

$$
|c| \leq 1-\frac{1}{\Re \delta} \sum_{i=1} \cdot \frac{2 M+1}{\left|\gamma_{i}\right|}
$$

and $\left|\mathcal{D}_{\lambda, \mu}^{m}\left(\alpha_{1}, \beta_{1}\right) f_{i}(z)\right| \leq M$ for all $i=1,2, \ldots, n$ and $z \in \Delta$, then the function $I_{\gamma_{i}, \delta}\left(\lambda, \mu, m ; \alpha_{1}, \beta_{1} ; z\right)$ defined by (1.3) is univalent.

Proof. Let

$$
h(z)=\int_{0}^{z}\left(\prod_{i=1}^{n} \frac{\mathcal{D}_{\lambda, \mu}^{m}\left(\alpha_{1}, \beta_{1}\right) f_{i}(t)}{t}\right)^{\frac{1}{\gamma_{i}}} d t
$$

so that we have

$$
h^{\prime}(z)=\left(\prod_{i=1}^{n} \frac{\mathcal{D}_{\lambda, \mu}^{m}\left(\alpha_{1}, \beta_{1}\right) f_{i}(z)}{z}\right)^{\frac{1}{\gamma_{i}}}
$$

and hence

$$
\frac{z h^{\prime \prime}(z)}{h^{\prime}(z)}=\sum_{i=1}^{n} \frac{1}{\gamma_{i}}\left(\frac{z\left(\mathcal{D}_{\lambda, \mu}^{m}\left(\alpha_{1}, \beta_{1}\right) f_{i}(z)^{\prime}\right.}{\mathcal{D}_{\lambda, \mu}^{m}\left(\alpha_{1}, \beta_{1}\right) f_{i}(z)}-1\right) .
$$

Consider

$$
\begin{aligned}
\left.|c| z\right|^{2 \delta}+ & \left(1-|z|^{2 \delta}\right) \frac{z h^{\prime \prime}(z)}{\delta h^{\prime}(z)} \mid \\
= & \left.|c| z\right|^{2 \delta}+\left(1-|z|^{2 \delta}\right) \frac{1}{\delta} \sum_{i=1}^{n}\left(\frac{z\left(\mathcal{D}_{\lambda, \mu}^{m}\left(\alpha_{1}, \beta_{1}\right) f_{i}(z)^{\prime}\right.}{\mathcal{D}_{\lambda, \mu}^{m}\left(\alpha_{1}, \beta_{1}\right) f_{i}(z)}-1\right) \mid \\
\leq|c| & +\frac{1}{\delta} \sum_{i=1}^{n} \frac{1}{\left|\gamma_{i}\right|}\left(\left|\frac{z^{2}\left(\mathcal{D}_{\lambda, \mu}^{m}\left(\alpha_{1}, \beta_{1}\right) f_{i}(z)\right)^{\prime}}{\left[\mathcal{D}_{\lambda, \mu}^{m}\left(\alpha_{1}, \beta_{1}\right) f_{i}(z)\right]^{2}}\right| \frac{\left|\mathcal{D}_{\lambda, \mu}^{m}\left(\alpha_{1}, \beta_{1}\right) f_{i}(z)\right|}{|z|}+1\right) .
\end{aligned}
$$

Since $\left|\mathcal{D}_{\lambda, \mu}^{m}\left(\alpha_{1}, \beta_{1}\right) f_{i}(z)\right| \leq M$ for $z \in \Delta$ and for $i=1,2, \ldots, n$, we have

$$
\left|\mathcal{D}_{\lambda, \mu}^{m}\left(\alpha_{1}, \beta_{1}\right) f_{i}(z)\right| \leq M|z|
$$


for $z \in \Delta$ and for $i=1,2, \ldots, n$. In view of (2.1) we get

$$
\left.|c| z\right|^{2 \delta}+\left(1-|z|^{2 \delta}\right) \frac{z h^{\prime \prime}(z)}{\delta h^{\prime}(z)}|\leq| c \mid+\frac{1}{\Re \delta} \sum_{i=1}^{n} \frac{2 M+1}{\left|\gamma_{i}\right|} \quad(z \in \Delta) .
$$

Hence by (3.1) we get

$$
\left.|c| z\right|^{2 \delta}+\left(1-|z|^{2 \delta}\right) \frac{z h^{\prime \prime}(z)}{\delta h^{\prime}(z)} \mid \leq 1 .
$$

Finally applying Theorem 2.3 we get the required result.

We remark here that by specializing the parameters involved in Theorem 3.1 we may arrive at the results in $[7,6,22,12,18]$.

\section{Another criteria for Univalence}

In this section we establish another criterion for univalence of the integral operator $\left.I_{\gamma_{i}, \delta}\left(\lambda, \mu, m ; \alpha_{1}, \beta_{1} ; z\right)\right)$ in the unit disk $\Delta$.

Theorem 4.1. Let the functions $\mathcal{D}_{\lambda, \mu}^{m}\left(\alpha_{1}, \beta_{1}\right) f_{i}(z) \in \mathcal{S}$ for $i=1,2, \ldots, n$, and $\delta$ be a complex number with $\Re \delta>0$. If

$$
\frac{1}{\left|\gamma_{1}\right|}+\frac{1}{\left|\gamma_{2}\right|}+\ldots+\frac{1}{\left|\gamma_{n}\right|} \leq \frac{1}{4}
$$

then the function $I_{\gamma_{i}, \delta}\left(\lambda, \mu, m ; \alpha_{1}, \beta_{1} ; z\right)$ is univalent.

Proof. Let $h(z)$ be defined as in Theorem 3.1, then

$$
\frac{z h^{\prime \prime}(z)}{h^{\prime}(z)}=\sum_{i=1}^{n} \frac{1}{\gamma_{i}}\left(\frac{z\left(\mathcal{D}_{\lambda, \mu}^{m}\left(\alpha_{1}, \beta_{1}\right) f_{i}(z)^{\prime}\right.}{\mathcal{D}_{\lambda, \mu}^{m}\left(\alpha_{1}, \beta_{1}\right) f_{i}(z)}-1\right) .
$$

Consider

$$
\begin{aligned}
\frac{1-|z|^{2 \Re \delta}}{\Re \delta}\left|\frac{z h^{\prime \prime}(z)}{h^{\prime}(z)}\right| & =\frac{1-|z|^{2 \Re \delta}}{\Re \delta} \sum_{i=1}^{n} \frac{1}{\left|\gamma_{i}\right|}\left|\frac{z\left(\mathcal{D}_{\lambda, \mu}^{m}\left(\alpha_{1}, \beta_{1}\right) f_{i}(z)\right)^{\prime}}{\mathcal{D}_{\lambda, \mu}^{m}\left(\alpha_{1}, \beta_{1}\right) f_{i}(z)}-1\right| \\
& \leq \frac{1-|z|^{2 \Re \delta}}{\Re \delta} \sum_{i=1}^{n} \frac{1}{\left|\gamma_{i}\right|}\left(\left|\frac{z\left(\mathcal{D}_{\lambda, \mu}^{m}\left(\alpha_{1}, \beta_{1}\right) f_{i}(z)\right)^{\prime}}{\mathcal{D}_{\lambda, \mu}^{m}\left(\alpha_{1}, \beta_{1}\right) f_{i}(z)}\right|+1\right) \\
& \leq \frac{1-|z|^{2 \Re \delta}}{\Re \delta} \sum_{i=1}^{n} \frac{1}{\left|\gamma_{i}\right|}\left(\frac{1+|z|}{1-|z|}+1\right)
\end{aligned}
$$




$$
=\frac{1-|z|^{2 \Re \delta}}{1-|z|} \frac{2}{\Re \delta} \sum_{i=1}^{n} \frac{1}{\left|\gamma_{i}\right|} .
$$

Noting the fact that

$$
\frac{1-|z|^{2 \Re \delta}}{1-|z|} \leq\left\{\begin{array}{lll}
1 & \text { if } & 0<\Re \delta<\frac{1}{2} \\
2 \Re \delta & \text { if } \quad & \frac{1}{2}<\Re \delta<\infty .
\end{array}\right.
$$

Using the inequality (4.1) and the hypothesis of the theorem we get

$$
\frac{1-|z|^{2 \Re \delta}}{\Re \delta}\left|\frac{z h^{\prime \prime}(z)}{h^{\prime}(z)}\right| \leq 1 \text {. }
$$

Hence the result follows as an application of Theorem 2.2.

Finally we remark that by specializing the parameters involved in Theorem 4.1 , the results in $[17,22]$ may be obtained as a special case of the Theorem 4.1 .

\section{References}

[1] Afaf. A. Ali Abubaker, M. Darus, Neighbourhoods of certain classes of analytic functions defined by a generalized differential operator, Int. J. Math. Anal., 4, No. 28 (2010), 2373-2380.

[2] R. Aghalary, S. B. Joshi, R. N. Mohapatra, V. Ravichandran, Subordinations for analytic functions defined by Dziok-Srivatsava linear operator, Appl. Math. Comput., 187, No. 1 (2007), 13-19.

[3] F. M. Al-Oboudi, On the univalent functions defined by a generalized Sălăgean operator, Int. J. Math. Math. Sci, 25-28 (2004), 1429-1436.

[4] D. Breaz, N. Breaz, Two integral operators, Studia. Univ. Babes-Bolyai. Mat., 47, No. 3 (2002), 13-19.

[5] D. Breaz, H. Ö. Güney, The integral operator on the classes $\mathcal{S}_{\alpha}^{*}(b)$ and $\mathcal{C}_{\alpha}(b)$, J. Math. Inequal., 2, No. 1 (2008), 97-100.

[6] D. Breaz, H. Ö. Güney, On the univalence criterion of a general integral operator, J. Inequal. Appl., Article ID 702715, 8 (2008).

[7] D. Breaz, N. Breaz, H. M. Srivatsava, An extension of univalent condition for a family of operators, Appl. Math. Lett., 22, No. 1 (2009), 41-44. 
[8] S. Bulut, Sufficient conditions for univalence of an integral operator defined by Al-Oboudi differential operator, J. Inequal. Appl., Article ID. 957042, 5 (2008).

[9] B. C. Carlson, S. B. Shaffer, Starlike and Prestarlike hypergeometric functions, SIAM. J. Math. Anal., 15(2002), 737-745.

[10] J. Dziok, H. M. Srivatsava, Classes of analytic functions associated with the generalized hypergeometric function, Appl. Math. Comput. 103, No. 1 (1999), 1-13.

[11] N Marikkannan, A subclass of analytic functions and a generalised differential operator, communicated.

[12] G. I. Oros, G. Oros, D. Breaz, Sufficient condition for univalence for an integral operator, J. Inequal. Appl., Article ID 127645, 7 (2008).

[13] S. Owa, Some applications of the fractional calculus, Fractional Calculus, Proc. Workshop, Ross Priory, Univ. Strathclyde/Engl. 1984 Res. Notes. Math.138 (1985), 164-175.

[14] S. Ozaki, M. Nunokawa, The Schwarzian derivative and univalent functions, Proc. Amer. Math. Soc., 33 (1972), 392-394.

[15] N. N. Pascu, On a univalence criterion (II), International seminar on functional equations, approximation and convexity, Cluj-Napoca, Preprint Nr.6 (1985), 153-154.

[16] V. Pescar, A new generalization of Ahlfors's and Becker's criterion of univalence, Bull. Malaysian. Math. Soc, 19, No. 2 (1996), 53-54.

[17] V. Pescar, New Univalence criterion for certain integral operator, Studia. Univ. Babeş-Bolyai. Math. Soc., 46, No. 2 (2001), 107-109.

[18] V. Pescar, On the univalence of some integral operators, J. Indian. Acad. Math., 27, No. 2 (2005), 239-243.

[19] St. Ruscheweyh, New criteria for univalent functions, Proc. Amer. Math. Soc., 49 (1975), 109-115.

[20] G. Ş. Sălăgean, Subclasses of univalent functions, in Complex analysisfifth Romanian-Finnish seminar, Part 1 (Bucharest, 1981), 362-372, Lecture Notes in Math., 1013 Springer, Berlin. 
[21] C. Selvaraj, K. R.Karthikeyan, Certain classes of analytic functions of complex order involving a family of generalized differential operator, $J$. Math. Ineq., 2, No. 4 (2008), 449-458.

[22] C. Selvaraj, K. R. Karthikeyan, Univalence of a generalized operator associated with the generalized hypergeometric function. Tamsuioxford J. Math. Sci., 26, No. 1 (2010), 41-51. 
